\title{
Leporidae Fischer, 1817 (Mammalia: Lagomorpha) de um depósito Quaternário do Brasil: comentários taxonômicos e tafonômicos
}

\section{Leporidae Fischer, 1817 (Mammalia: Lagomorpha) from a Quaternary deposit in Brazil: taxonomic and taphonomic comments}

\author{
Artur Chahud' (1) | Gabriela Sartori Mingatos', "I (1) | Mercedes Okumural \\ Universidade de São Paulo. Laboratório de Estudos Evolutivos Humanos. São Paulo, São Paulo, Brasil \\ "Museu Nacional. Universidade Federal do Rio de Janeiro. Programa de Pós-Graduação em Arqueologia. Rio de Janeiro, Rio de Janeiro, Brasil
}

Resumo: Leporidae (Lagomorpha) é um clado de pequenos mamíferos que inclui os coelhos e as lebres, animais raros em sítios
paleontológicos e arqueológicos e, por isso, leporídeos são pouco estudados na América do Sul. Durante o Quaternário,
muitas cavernas calcárias serviram como armadilhas naturais para inúmeros grupos de animais, incluindo leporídeos. A
Gruta Cuvieri, parte do complexo cárstico de Lagoa Santa (Minas Gerais, Brasil), preservou grande quantidade de restos
desses animais. O presente estudo descreve o material osteológico (dentários, maxilas e alguns ossos apendiculares)
atribuído a Sylvilagus cf. brasiliensis, encontrado na Gruta Cuvieri, além de revisar sua ocorrência durante o Quaternário
e comparar as datações obtidas com a frequência de ocorrências desse táxon em sítios paleontológicos e arqueológicos.
Os espécimes estavam presentes no Pleistoceno final, reaparecendo no Holoceno médio e persistindo até recentemente.

Palavras-chave: Gruta Cuvieri. Holoceno. Lagoa Santa. Paleoambiente. Pleistoceno. Sylvilagus.

\begin{abstract}
Leporidae (Lagomorpha) is a clade of small mammals that include rabbits and hares, rarely found in paleontological and archaeological sites and, therefore, leporids are poorly studied in South America. During the Quaternary, many limestone caves worked as natural traps for several groups of animals, including Leporidae. The Cuvieri Cave, part of the Lagoa Santa (Minas Gerais state, Brazil) karst complex, preserved a large amount of the remains of these animals. The present study describes the osteological material (mandibles, jaws and some appendicular bones) assigned to Sylvilagus cf. brasiliensis, which were found in the Cuvieri Cave. In addition, we reviewed the occurrences of these animals in Quaternary sites and compared the radiometric ages obtained with the frequency of occurrences of this taxon. The specimens were present in the late Pleistocene, reappearing in the middle Holocene and persisting until recently.
\end{abstract}

Keywords: Cuvieri Cave. Holocene. Lagoa Santa. Paleoenviroment. Pleistocene. Sylvilagus.

CHAHUD, A., G. S. MINGATOS \& M. OKUMURA, 2020. Leporidae Fischer, 1817 (Mammalia: Lagomorpha) de um depósito Quaternário do Brasil: comentários taxonômicos e tafonômicos. Boletim do Museu Paraense Emílio Goeldi. Ciências Naturais 15(3): 795-806. DOI: http://doi.org/10.46357/bcnaturais.v15i3.299.

Autor para correspondência: Artur Chahud. Universidade de São Paulo. Instituto de Biociências. Departamento de Genética e Biologia Evolutiva. Laboratório de Estudos Evolutivos Humanos (LEEH). Rua do Matão, 277. São Paulo, SP, Brasil. CEP $05508-090$ (arturchahud@yahoo.com).

Recebido em 11/04/2020

Aprovado em 27/11/2020

Responsabilidade editorial: Adriano Oliveira Maciel e Alexandra Maria Ramos Bezerra
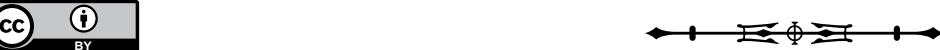


\section{INTRODUÇÃO}

Os registros mais antigos de Lagomorpha são do Eoceno da Índia (Rose et al., 2008), porém foi durante o Oligoceno que espécies semelhantes aos atuais Leporidae surgiram na América do Norte (Emry \& Gawne, 1986). Apesar de ocorrerem durante boa parte do Cenozoico, os registros mais antigos na América do Sul são do final do Pleistoceno, da região pré-andina do Equador, Formação Cangahua (Ficcarelli et al., 1992), durante o quarto Grande Intercâmbio Biótico Americano (GABI 4), ocorrido em torno de 125 mil anos atrás (Woodburne, 2010).

No Brasil, a ocorrência de fósseis é bastante escassa. As primeiras citações são da região do Rio das Velhas, estado de Minas Gerais, coletadas por Peter Lund e estudadas por Winge (1887), que considerou a espécie encontrada como pertencente ao gênero Sylvilagus Gray, 1867, como uma variedade pleistocênica de Sylvilagus brasiliensis Linnaeus, 1758, de tamanho um pouco maior. Outro registro foi feito por Ameghino (1907), para o município de Iporanga, estado de São Paulo, porém a revisão desse material, realizada por Paula Couto (1979), resultou na proposta de que tais espécimes pertenceriam à espécie atual, S. brasiliensis.
A Gruta Cuvieri, localizada no Carste de Lagoa Santa, estado de Minas Gerais, Brasil, é conhecida por possuir grande quantidade de material osteológico quaternário (Hubbe et al., 2011; Chahud, 2020a, 2020b, 2020c; Chahud \& Okumura, 2020a, 2020b), incluindo material de Leporidae. Contudo, as ocorrências em sítios arqueológicos da mesma região são raras ou pouco registradas (Kipnis, 2002; Mingatos \& Okumura, 2016; Mingatos, 2017; Chim, 2018).

O objetivo desta contribuição é reportar a ocorrência de Leporidae na Gruta Cuvieri e revisar ocorrências em sítios paleontológicos e arqueológicos, acrescentando informações sobre esse grupo, pouco conhecido do Quaternário do Brasil.

\section{MATERIAL E MÉTODOS}

A Gruta Cuvieri, localizada no município de Lagoa Santa, centro-sul do estado de Minas Gerais, Brasil, coordenadas UTM 23k 7846105N e 0603756E, é constituída de três pequenas cavidades verticais, armadilhas naturais que foram denominadas Loci 1, 2 e 3 (Figura 1), com 16 metros, 4 metros e 8 metros de profundidade, respectivamente. O material osteológico foi coletado com rigoroso detalhamento estratigráfico dos Loci 2 e 3 (Hubbe et al., 2011;

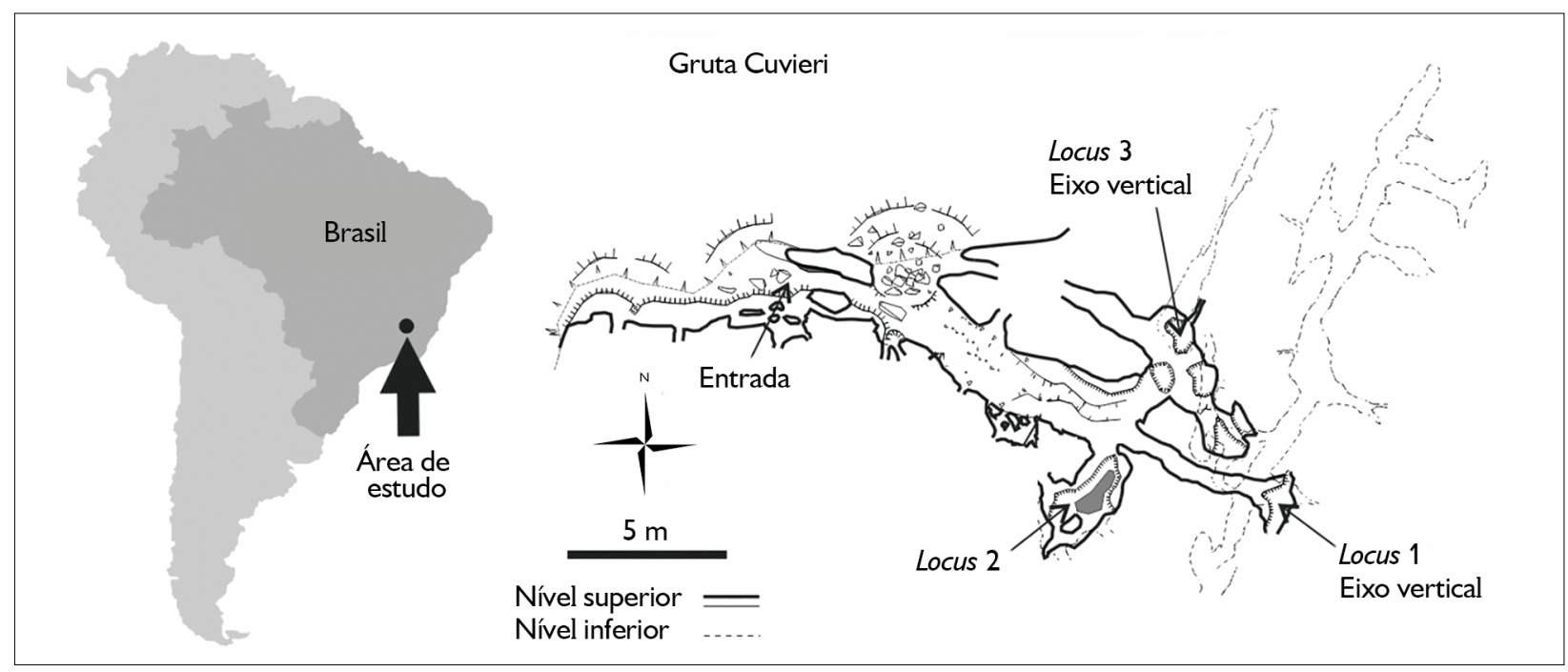

Figura 1. Localização geográfica da área de estudo no Brasil (esquerda) e da Gruta Cuvieri mostrando a posição do Loci 1, 2 e 3 (direita). Mapa produzido por Alex Hubbe e Grupo Bambuí de Pesquisas Espeleológicas para o Laboratório de Estudos Evolutivos Humanos.

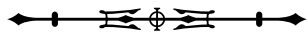


Haddad-Martim et al., 2017). Segundo Hubbe (2008), Hubbe et al. (2011), Mayer (2011) e Haddad-Martim et al. (2017), o sedimento encontrado na Gruta Cuvieri estava pouco consolidado, remobilizado ou modificado por fatores intempéricos externos, o que favoreceu a escavação e a exploração mais cuidadosa, com obtenção de dados estratigráficos detalhados.

Os aspectos tafonômicos, como definição de número de indivíduos, abrasão ou desgaste, nível de exposição, quebras, ataque de carniceiros ou predadores, presença de epífises e articulação, foram os principais itens avaliados, seguindo os conceitos de Voorhies (1969), Badgley (1986), Behrensmeyer (1991) e Lyman (1994). Para a identificação dos espécimes, foi feita a comparação com aqueles da Coleção de Referência "Renato Kipnis" do Laboratório de Estudos Evolutivos Humanos do IB-USP, além de consulta às obras de Paula Couto (1979), Ruedas (2017), Ruedas et al. (2017) e Diersing \& Wilson (2017). material osteológico coletado referente à Gruta Cuvieri é constituído de 82 elementos ósseos (excluindo dentes isolados), sendo 46 do Locus 2 e 36 do Locus 3. O material é constituído de fragmentos cranianos, ossos apendiculares e poucas vértebras. Os espécimes e o inventário de suas partes ósseas estão depositados no Laboratório de Estudos Evolutivos Humanos (LEEH) do Instituto de Biociências da Universidade de São Paulo (IB-USP), São Paulo, Brasil.

\section{CONSIDERAÇÕES SOBRE IDADE E PALEOAMBIENTE}

Não foi possível a datação dos espécimes encontrados, no entanto Hubbe (2008) e Hubbe et al. (2011) obtiveram inúmeras datações de diversos grupos animais no Locus 2 e de espeleotema e fauna do Locus 3. No total, de acordo com os dados reportados por esses autores, 17 datações foram realizadas no Locus 2 (Tabela 1),

Tabela 1. Datações 14C-AMS (AMS = Accelerator Mass Spectrometry) obtidas no Locus 2 da Gruta Cuvieri, Lagoa Santa, estado de Minas Gerais (Hubbe et al., 2011). Abreviações: $m=$ metros, AP = anos antes do presente.

\begin{tabular}{|c|c|c|c|c|}
\hline Espécime & Táxons & Profundidade (m) & Idade convencional (AP) & Calibração $2 \sigma(A P)$ \\
\hline CVL2-P260 & Cervidae & 0,62 & $1.960 \pm 40$ & $1.990-1.830$ \\
\hline CVL2-P163/196 & Tapirus of. terrestris & 0,62 & $2.050 \pm 40$ & $2.120-1.900$ \\
\hline CVL2-P258/234 & Cuniculus paca & 0,62 & $220 \pm 40$ & $310-0$ \\
\hline CVL2-2290 & Cervidae & 0,69 & $2.830 \pm 40$ & $3.060-2.850$ \\
\hline CV-L2-4041 & Amphibia & 0,73 & $3.550 \pm 40$ & $3.960-3.710$ \\
\hline CVL2-4630 & Tayassuidae & 0,76 & $5.250 \pm 50$ & $6.180-5.920$ \\
\hline CVL2-5998 & Cuniculus paca & 0,80 & $5.050 \pm 40$ & $5.910-5.670$ \\
\hline CVL2-7108 & Cervidae & 0,86 & $5.200 \pm 50$ & $6.010-5.900$ \\
\hline CVL2-7402 & Cervidae & 0,86 & $9.500 \pm 50$ & $11.070-10.660$ \\
\hline CVL2-7456 & Tayassuidae & 0,86 & $5.150 \pm 50$ & $5.990-5.750$ \\
\hline CV-L2-8040 & Cervidae & 0,87 & $7.050 \pm 50$ & $7.970-7.790$ \\
\hline CVL2-9648 & Cervidae & 0,94 & $9.740 \pm 40$ & $11.210-11.130$ \\
\hline CVL2-10365 & Cervidae & 0,96 & $6.930 \pm 40$ & $7.850-7.680$ \\
\hline CV-L2-13122 & Smilodon populator & 1,11 & $10.790 \pm 60$ & $12.880-12.720$ \\
\hline CV-L2 14310 & Valgipes bucklandi & 1,14 & $11.020 \pm 40$ & $13.060-12.870$ \\
\hline CV-L2 14827 & Cervidae & 1,16 & $7.690 \pm 50$ & $8.580-8.400$ \\
\hline CV-L2 15266 & Tayassuidae & 1,20 & $10.470 \pm 40$ & $12.670-12.140$ \\
\hline
\end{tabular}


todas utilizando a técnica de 14C-AMS (Accelerator Mass Spectrometry) (Hubbe et al., 2011). Observando as datações, é perceptível que a maior parte delas (total de 12) seguia uma estratigrafia organizada, sendo o material mais antigo encontrado nas camadas mais profundas. Os espécimes coletados no Locus 2 estão bem distribuídos e posicionados, se fazendo presentes desde a superfície até 0,80 m de profundidade (Tabela 1). Nesses níveis, foram estimadas idades que não superaram 6.180 anos AP (antes do presente) (datações calibradas).

O Locus 3 possui poucas datações e, mesmo que existissem outras, a associação entre restos faunísticos e datações seria mais difícil, pois os ossos ocorrem desde a superfície até a parte mais profunda escavada, estando assim espalhados por todo o pacote sedimentar. No Locus 3 , foram obtidas duas datações utilizando a técnica de 14C-AMS: uma de $12.390 \pm 50$ AP para um espécime de Tapiridae e outra de $12.510 \pm 70$ AP para uma preguiça terrestre, sendo que o primeiro estava próximo da superfície. Além das datações citadas, existem três datações realizadas com a técnica de U/Th (Urânio/Tório) que obtiveram valores entre 27 e 31 mil anos a partir de análise de material obtido em capa estalagmítica (Hubbe, 2008).

A sedimentação nos dois loci é diferente. Segundo Hubbe (2008), o aporte de sedimento no Locus 3 é diferenciado do Locus 2, na medida em que no primeiro ocorre grande variedade de fácies, verticalmente e horizontalmente, enquanto no segundo a sedimentação é homogênea. Esta característica pode ter favorecido maior remobilização de material no Locus 3, se compararmos com o Locus 2, causando a mistura do material osteológico de diferentes idades. Considerando a menor remobilização de sedimento e seus conteúdos no Locus 2, em comparação com o Locus 3, o levantamento estratigráfico mostrou-se suficientemente confiável para associação dos materiais com as idades propostas. No entanto, por ser um depósito em caverna, essa associação deve ser feita com cautela, pois ocorre remobilização de ossos menores que, em geral, é maior do que a de ossos maiores ou mais pesados (Voorhies, 1969; Behrensmeyer, 1991), colocando no mesmo nível material ósseo de diferentes idades.

Partindo dessas observações e de que os Leporidae surgiram naAmérica do Sul ainda no Pleistoceno (Woodburne, 2010), é possível inferir que foram mais comuns na região da Gruta Cuvieri em torno de 5.000-6.000 AP, baseando-se nas ocorrências do Locus 2. Porém, não se deve descartar um período de abundância e presença no Pleistoceno devido às ocorrências no Locus 3, observando que as datações obtidas neste locus são pleistocênicas. Estudos paleoambientais realizados na região de Lagoa Santa apontaram para um clima úmido ao longo do Holoceno inicial (11.700-8.200 AP), seguido por um período seco que se teria iniciado por volta de 8.000 AP (Araujo et al., 2005). No entanto, Hermenegildo (2009) considera que, na região de Lagoa Santa, a pluviosidade teria aumentado a 6.200 AP. Esse último dado estaria de acordo com o esperado para um clima que favorecesse o reaparecimento dos Leporidae na Gruta Cuvieri em torno de cinco e seis mil anos.

\section{PALEONTOLOGIA SISTEMÁTICA}

Mammalia Linnaeus, 1758

Lagomorpha Brandt, 1855

Leporidae Fischer von Waldheim, 1817 Sylvilagus Gray, 1867

Sylvilagus of. brasiliensis Linnaeus, 1758

(Figuras 2-3)

Material estudado do Locus 2: o material utilizado para a identificação inclui quatro dentários (CVL2-4922, CVL2-3694, CVL2-P2879, CVL2-P712), cinco maxilas (CVL2-4162, CVL2-4252, CVL2-4253, CVL2-P184, CVL2-P316), dois fêmures (CVL2-4956, CVL2-591), três tíbias (CVL2-2552, CVL2-4752, CVL2-1156), uma ulna (CVL2-5570), cinco calcâneos (CVL2-P3731, CVL2-P3396, CVL2-4757, CVL2-P200, CVL2-P2880), uma escápula (CVL2-P3408) e três úmeros (CVL2-3097, CVL2-P3692, CVL2-P3693).

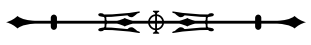


Material estudado do Locus 3: o material utilizado para a identificação inclui dez dentários (CVL3-2024, CVL33362, CVL3-13, CVL3-2754, CVL3-P1505, CVL3-P126, CVL3-P9530, CVL3-3801, CVL3-P938, CVL3-10367), uma maxila (CVL3-P10318), quatro fêmures (CVL3-4225, CVL3-7A, CVL3-1489, CVL3-4226), quatro tíbias (CVL3369, CVL3-1976, CVL3-2025, CVL3-4236), três rádios (CL3-4A, CVL3-6A, CVL3-4171), uma ulna (CVL3-6B) e três úmeros (CVL3-3425, CVL3-2213, CVL3-1977).

Distribuição geográfica e ambiental: espécie típica de florestas tropicais úmidas do sul do México ao norte da Argentina e Sul do Brasil (Hoffman \& Smith, 2005).

Comentários: a taxonomia dos representantes de Sylvilagus na América do Sul é controversa, não havendo consenso entre os trabalhos publicados. Para Hoffman \& Smith (2005), apenas uma espécie, Sylvilagus brasiliensis Linnaeus, 1758, era válida e os autores reconheciam ainda mais 21 subespécies, porém Ruedas (2017) reconhece sete espécies atribuídas ao gênero Sylvilagus na América do Sul: Sylvilagus brasiliensis Linnaeus, 1758, S. tapetillus Thomas, 1913, S. floridanus J. A. Allen, 1890, S. andinus Thomas, 1897, S. sanctaemartae Hershkovitz, 1950, S. varynaensis Durant \& Guevara, 2001 e S. parentum Ruedas, 2017. As espécies $S$. andinus, S. floridanus, S. sanctaemartae, $S$. varynaensis e $S$. parentum ocorrem localmente, sendo as quatro primeiras observadas em regiões dos Andes, e S. parentum está restrita à Guiana (Ruedas, 2017). A classificação de Ruedas (2017) não foi aceita por Diersing \& Wilson (2017), que consideraram Sylvilagus sanctaemartae como sinônimo de S. gabbi J. A. Allen, 1877, da América Central, e Sylvilagus andinus como subespécie de $S$. brasiliensis, considerada S. b. andinus.

Entre todas as subespécies e espécies citadas anteriormente, apenas duas, Sylvilagus brasiliensis e $S$. tapetillus, ocorrem no Brasil. A espécie S. tapetillus foi reconhecida a partir de três indivíduos coletados na região do Vale do Paraíba, no município de Resende, estado do Rio de Janeiro. Segundo Bonvicino et al. (2015) e Ruedas et al. (2017), a espécie é diagnóstica tanto com base na morfologia externa, quanto pelos dados genéticos. Os espécimes da Gruta Cuvieri puderam ser classificados com base nos poucos dentários recuperados (Figura 2). A dentição e o formato são características da família Leporidae e podem ser atribuídas ao gênero Sylvilagus (Paula Couto, 1979; Ruedas et al., 2017).

Comparando com Sylvilagus tapetillus, os dentários são mais alongados, finos e gráceis dorsoventralmente e o forame mentoniano é característico de Sylvilagus brasiliensis em forma, tamanho e posição (Figuras 2A, 2D e 2F). Segundo Ruedas et al. (2017), há variação no tamanho do forame mentoniano em S. brasiliensis, porém é maior e mais desenvolvido em $S$. tapetillus. Embora os espécimes da Gruta Cuvieri apresentem ossos pós-cranianos, esses não são informativos para a identificação de espécie. Não foram preservadas outras partes cranianas, portanto, a identificação taxonômica foi baseada apenas nos dentários. Por esse motivo, optouse por atribuir os espécimes a Sylvilagus cf. brasiliensis. Assim, espécimes mais completos são necessários para uma identificação taxonômica mais segura.

\section{TAFONOMIA}

As observações dos ossos pós-cranianos revelaram diferenças de tamanho de alguns indivíduos, porém não havia filhotes recém-nascidos ou indivíduos muito jovens que poderiam caracterizar que a Gruta Cuvieri foi utilizada como habitação e proteção segura para a reprodução. Evidências da presença de indivíduos jovens aparecem na forma de um fêmur, uma tíbia (Figuras 3A e 3B) e uma epífise de fêmur não fusionada no Locus 2 , porém eram formas adolescentes e, pelas proporções, poderiam pertencer ao mesmo indivíduo.

Todos os espécimes estavam desarticulados e com várias quebras e desgastes (Figuras 3A, 3C e 3D), considerando que houve remobilização de diversas partes ósseas e os ossos menores e mais frágeis foram perdidos. Esse dado é importante devido ao fato de não ter sido observada essa perda e desarticulação de 


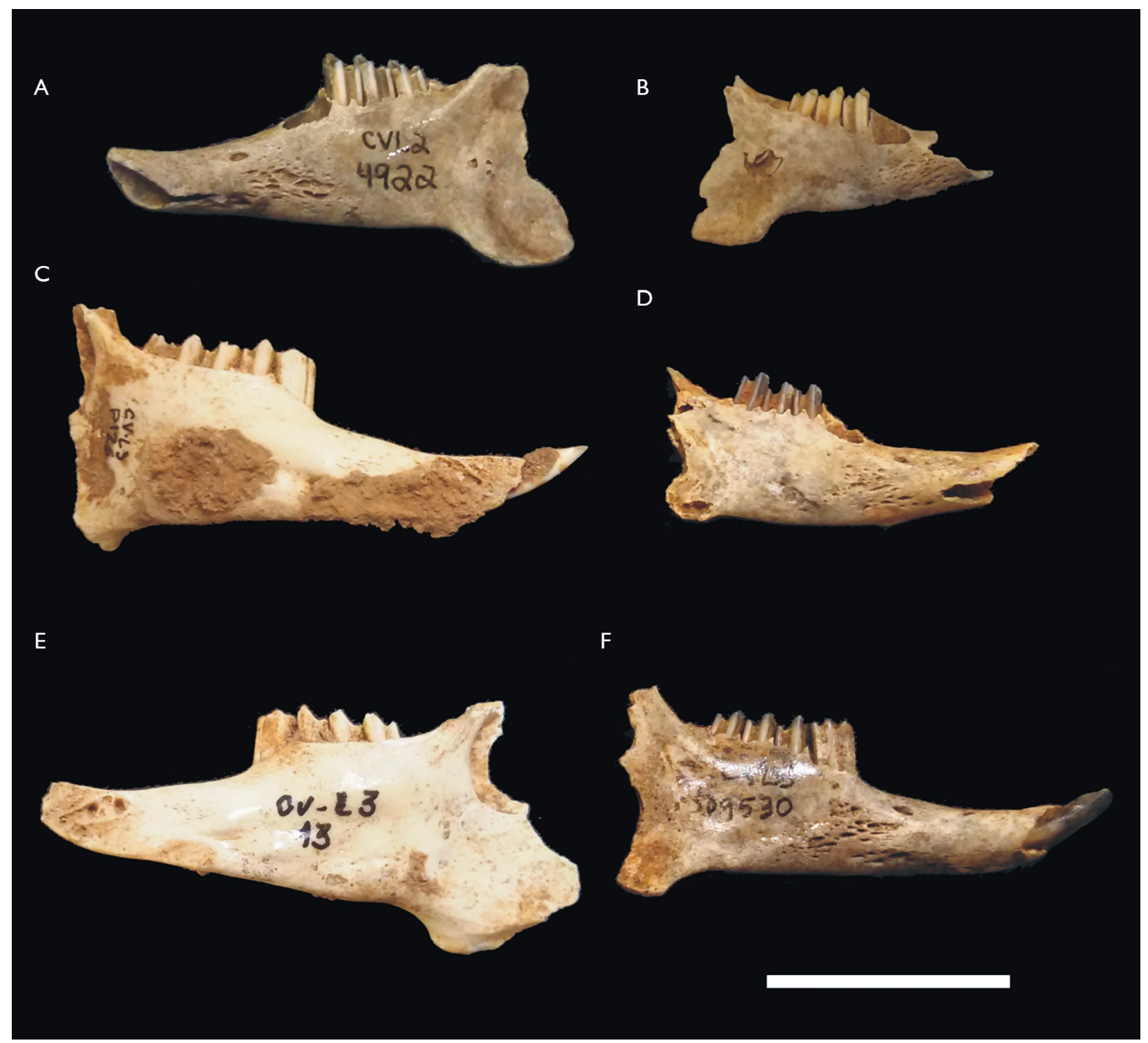

Figura 2. Dentários de Sylvilagus cf. brasiliensis: A-B) dentários provenientes do Locus 2 (CVL2-4922, CVL2-2879); C-F) dentários encontrados no Locus 3 (CVL3-P126, CVL3-P3801, CVL3-13, CVL3-P9530). Vistas laterais. Escala: 20 mm. Fotos: Artur Chahud (2020).

forma frequente nos esqueletos de animais maiores (cervos, porcos, antas) da Gruta Cuvieri, na qual foram observadas diversas carcaças ainda articuladas ou em posição de morte (Hubbe et al., 2011). Dessa forma, é possível inferir que o tamanho dos espécimes teria sido um fator significativo na fragmentação e dispersão do material osteológico.
Aparentemente os espécimes de Sylvilagus cf. brasiliensis teriam caído nas armadilhas naturais formadas pelos abismos da Gruta Cuvieri, do mesmo modo que teria ocorrido com animais de maior tamanho (Hubbe, 2008; Mayer, 2011). Essa hipótese é suportada pelo fato de um espécime do Locus 3 (dentário ilustrado na Figura 2E) ter sido encontrado com os ossos

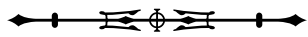




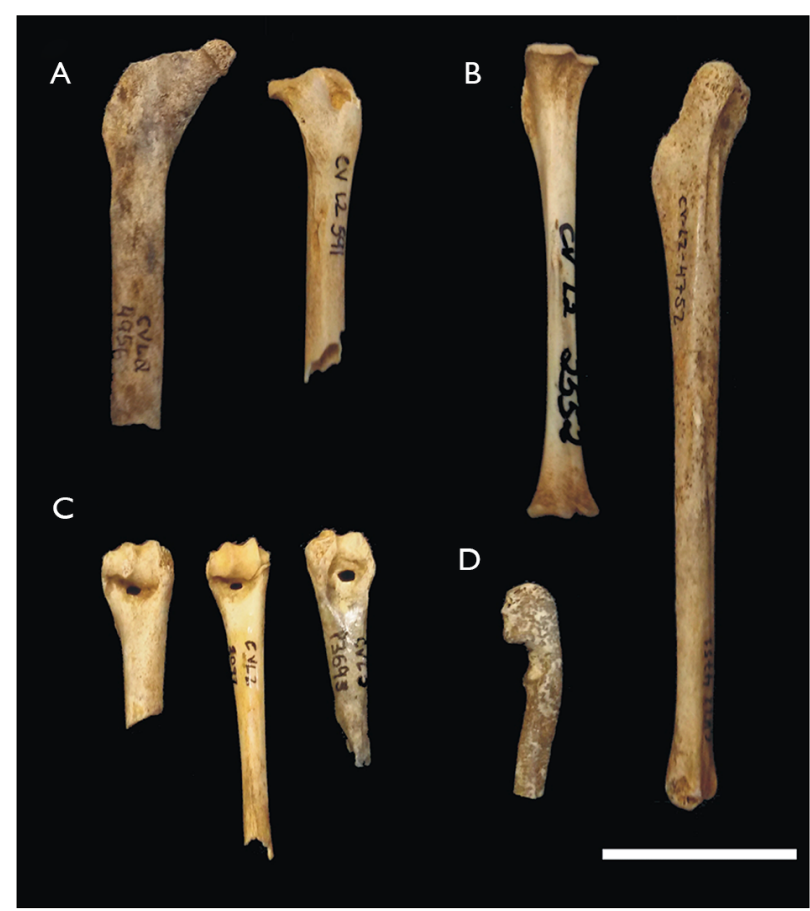

Figura 3. Ossos apendiculares de Sylvilagus cf. brasiliensis coletados do Locus 2: A) fêmur de um indivíduo adulto (CVL2-4956) ao lado de um jovem (CVL2-591), ambos com quebras e desgaste na superfície; B) tíbia de indivíduo jovem (CVL2-2552) ao lado de uma tíbia de adulto (CVL2-4752) com quebras e desgaste na extremidade; C) úmeros com quebras nas partes proximais (CVL2-P3692, CVL23097, CVL2-P3693); D) ulna fragmentada (CVL2-5570). Escala: 20 mm. Fotos: Artur Chahud (2020).

pós-cranianos associados na camada superior do locus. Além disso, devido ao tamanho diminuto das partes ósseas encontradas, dificilmente essas teriam suportado grandes períodos de transporte e retrabalhamento.

A partir do número de elementos ósseos coletados e reconhecidos nos dois loci, foi possível determinar o número de indivíduos, utilizando a técnica de mínimo número de indivíduos (MNI), baseada nos calcâneos e dentários do Locus 2 e nos dentários do Locus 3, que são os elementos mais abundantes. Assim, estimou-se que o número mínimo de indivíduos no Locus 2 seria de três indivíduos e no Locus 3, seis indivíduos. Observando as características homogêneas de deposição do Locus 2 , seria possível a definição do número de indivíduos baseada nas idades obtidas de outros espécimes datados. Porém, o material osteológico está restrito a $30 \mathrm{~cm}$ de espessura, camada muito pouco espessa e que poderia conter alguma mistura temporal de ossos menores, e também ao registro de algumas idades variadas (Hubbe et al., 2011), demonstrando idades maiores em níveis similares (Tabela 1). A determinação do número de indivíduos por níveis ou estratos não se mostrou segura ou confiável, observando que a sedimentação em caverna não era totalmente estável, contínua, sem perturbação biológica ou física, como esperado em ambiente de decantação sedimentar em fundo de lagos ou mares (Holz \& Simões, 2002).

A Tabela 2 apresenta os elementos de Sylvilagus cf. brasiliensis encontrados nos Loci 2 e 3 da Gruta Cuvieri. É possível observar que o Locus 2 tem maior número de elementos, embora apresente maior quantidade de partes ósseas muito frágeis, como vértebras (11 no Locus 2 e apenas uma no Locus 3). Já o Locus 3 possui partes esqueletais mais difíceis de serem perdidas, como dentários (dez no Locus 3 e apenas quatro no Locus 2).

No Locus 3, as partes frágeis existentes incluem três metacarpos, uma vértebra e uma ulna, que pertencem a um único indivíduo que estava próximo da superfície. Dadas a coloração esbranquiçada (Figura 2E) e a pouca abrasão observada, possivelmente deve ser um espécime mais recente e que não sofreu os processos físicos e biológicos locais por muito tempo. Ao todo, esse espécime é constituído por dez ossos que estavam associados e muito próximos entre si, representando quase $27,8 \%$ de todo o material do Locus 3.

Ocorrem dentes isolados em ambos os Loci, porém, no Locus 2, a quantidade é muito maior, com 27 elementos (muitos associados a dentários ou maxilas já citadas na Tabela 2 e, por isso, não foram contabilizados) contra três do Locus 3. Esse dado reforça que o ambiente deposicional do Locus 2 foi menos perturbado do que no Locus 3, possibilitando, assim, a preservação de pequenos ossos e de dentes. 
Tabela 2. Elementos esqueléticos coletados de espécimes de Sylvilagus encontrados nos Loci 2 e 3 da Gruta Cuvieri, Lagoa Santa, Minas Gerais, Brasil. Abreviações: $\mathrm{E}=$ esquerdo, $\mathrm{D}=$ direito, $\mathrm{I}=$ indeterminado, $\mathrm{N}=$ número de elementos.

\begin{tabular}{|c|c|c|c|}
\hline \multicolumn{2}{|c|}{ Locus 2} & \multicolumn{2}{|c|}{ Locus 3} \\
\hline Parte óssea & $N$ & Parte óssea & $N$ \\
\hline Calcâneo & 5 (2E e 3D) & Escápula & $2(2 E)$ \\
\hline Costela & 1 & Fêmur & $4(3 \mathrm{D}$ e $1 E)$ \\
\hline Epífise distal de fêmur & 1 & Dentário & 10 (4E e 6D) \\
\hline Escápula & $1(1 \mathrm{E})$ & Maxila & $1(1 \mathrm{E})$ \\
\hline Falange & 1 & Metacarpo & 3 \\
\hline Fêmur & $2(2 D)$ & Pélvis & $4(2 E$ e $2 D)$ \\
\hline Dentário & 4 (3D e 1E) & Rádio & 3 (2E e 1D) \\
\hline Maxila & $5(1 \mathrm{D}, 2 \mathrm{E}, 2 \mathrm{I})$ & Tíbia & $4(2 \mathrm{D}$ e $2 \mathrm{E})$ \\
\hline Metapódio & 3 & Ulna & $1(1 \mathrm{E})$ \\
\hline Metatarso & 1 & Úmero & $3(2 \mathrm{E}$ e $1 \mathrm{D})$ \\
\hline Pélvis & $3(3 D)$ & Vértebra & 1 \\
\hline Rádio & $1(1 \mathrm{D})$ & - & - \\
\hline Tíbia & 3 (1D e 2E) & - & - \\
\hline Ulna & $1(1 \mathrm{E})$ & - & - \\
\hline Úmero & $3(3 E)$ & - & - \\
\hline Vértebra & 11 & - & - \\
\hline Total & 46 & Total & 36 \\
\hline
\end{tabular}

\section{OCORRÊNCIAS EM SÍTIOS ARQUEOLÓGICOS}

$O$ registro de ocorrências de Leporidae em sítios arqueológicos é muito abundante no hemisfério norte, com evidência da caça desses animais em sítios Clovis (Waguespack \& Surovell, 2003) e pré-Clovis (Hockett \& Jenkins, 2013). A presença desses animais no registro arqueológico se explica possivelmente devido a esse grupo ser grande e diversificado na América do Norte. Na América do Sul, especialmente no Brasil, os registros são escassos e a ocorrência, muitas vezes, está restrita a um único osso ou fragmento (Kipnis, 2002). Além de Kipnis (2002), outros estudos mencionam a presença de Sylvilagus brasiliensis em sítios arqueológicos: śtio Garivaldino, município de Brochier, estado do Rio Grande do Sul (Queiroz, 2001; Rosa, 2009; Jacobus \& Rosa, 2013); śtio Água Limpa, em Monte Alto (Alves et al., 2005), e sambaqui fluvial Laranjal, em Miracatu (Borges, 2009), ambos no estado de São Paulo; e sambaqui costeiro do Moa, em Saquarema, estado do Rio de Janeiro (Imazio da Silveira, 2001). A Tabela 3 apresenta o MNI e o número de espécimes identificados (NISP) desse táxon nos sítios arqueológicos supracitados.

Aparentemente, devido à sua raridade (Tabela 3), os Sylvilagus não eram escolhas preferenciais dos grupos caçadores-coletores do Holoceno e, de acordo com o modelo proposto por Kipnis (2002), ocupariam o trigésimo lugar na preferência de caça desses grupos. No estado de Minas Gerais estão registrados, no Cadastro Nacional de Sítios Arqueológicos (CNSA), cerca de 1.300 sítios arqueológicos pré-coloniais. Desses, poucos possuem informações sobre composição faunística e apenas Lapa do Boquete (Kipnis, 2002), Lapa dos Bichos (Kipnis, 2002), Santana do Riacho (Kipnis, 2002), Lapa das Boleiras (Kipnis et al., 2010) apresentam informação sobre a presença de Sylvilagus brasiliensis (Tabela 3). Contudo, no que se refere

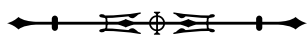


Tabela 3. Espécimes de Sylvilagus observados em sítios arqueológicos no Brasil. * = Datas inferidas a partir de Kipnis (2002). Abreviações: MNI = mínimo número de indivíduos, NISP = número de espécimes identificados, UF = unidade da federação, MG = Minas Gerais, $\mathrm{RJ}=$ Rio de Janeiro, RS = Rio Grande do Sul, SP = São Paulo, AP = anos antes do presente.

\begin{tabular}{c|c|c|c|c|c|c}
\hline Sítio & Município & UF & MNI & NISP & Idade convencional (AP) & Fonte \\
\hline Santana do Riacho & Vale do Peruaçu & MG & 3 & 8 & $<6.000 *$ & Kipnis (2002) \\
\hline Lapa dos Bichos & Vale do Peruaçu & MG & 3 & 3 & $<6.000^{*}$ e $>8.500 *$ & Kipnis (2002) \\
\hline Lapa do Boquete & Vale do Peruaçu & MG & 2 & 3 & $>9.000 *$ & Kipnis (2002) \\
\hline Sambaqui do Moa & Saquarema & RJ & 1 & 2 & $3.960 \pm 200$ & Imazio da Silveira (2001) \\
\hline Garivaldino & Brochier & RS & 1 & 2 & $7.520 \pm 350$ & Queiroz (2001) \\
\hline Garivaldino & Brochier & RS & 1 & 4 & $8.020 \pm 150$ & Queiroz (2001) \\
\hline Garivaldino & Brochier & RS & 1 & 3 & $8.290 \pm 130$ & Queiroz (2001) \\
\hline Garivaldino & Brochier & RS & 1 & 1 & $9.439 \pm 360$ & Queiroz (2001) \\
\hline Sambaqui Laranjal & Miracatu & SP & 2 & 2 & $6.890 \pm 90$ & Borges (2009) \\
\hline Sítio Água Limpa & Monte Alto & SP & - & - & $1.524 \pm 152$ & Alves et al. (2005) \\
\hline
\end{tabular}

ao sítio Lapa das Boleiras, o único fragmento ósseo obtido ao longo das escavações e identificado como Sylvilagus brasiliensis foi encontrado em uma das quadras localizada na porção norte do sítio. É sabido que nas quadras da parte norte desse sítio foram encontrados fragmentos de cerâmica, vidro e louça do século XIX, misturados a fragmentos de fauna e material lascado ao longo de um metro de profundidade. De acordo com Araujo et al. (2010), tais quadras (K25 e L25) foram datadas em $180 \pm$ $40 \mathrm{AP}$ e, por isso, não deve ser descartada a possibilidade de este espécime ser recente e doméstico.

São poucas as ocorrências de Sylvilagus brasiliensis em sítios arqueológicos brasileiros, porém é possível observar que as ocorrências ao sul do estado de Minas Gerais concentram-se no limite do Holoceno médio e Holoceno inicial (datas de Garivaldino e Laranjal), enquanto os sítios mineiros pesquisados por Kipnis (2002) possuem idades equivalentes às observadas no Locus 2 da Gruta Cuvieri, contudo também apresentam datações anteriores, do Holoceno inicial.

\section{CONCLUSÃO}

Os espécimes estudados foram encontrados em duas localidades (Locus 2 e Locus 3), que apresentam diferentes características deposicionais, sendo o Locus 2 de sedimentação mais homogênea, favorecendo a preservação de material osteológico menor e/ou mais frágil (vértebras, costelas, falanges e dentes). Devido à sedimentação homogênea e à pouca remobilização, o Locus 2 também pôde ser usado para fazer uma correlação entre idade e camada, através da qual foi possível sugerir que os espécimes encontrados não seriam mais antigos do que seis mil anos. Ao material osteológico do Locus 3 , baseado nas idades obtidas e na correlação estratigráfica, foi atribuída uma idade pleistocênica.

Os espécimes encontrados na Gruta Cuvieri foram identificados como Sylvilagus cf. brasiliensis, a partir de características morfológicas dos dentários e, por isso, essa identificação deve ser considerada com cautela. Todavia, dados os estudos recentes de revisão do gênero Sylvilagus, que propõem a presença de outras espécies atuais (Ruedas, 2017; Ruedas et al., 2017; Diersing \& Wilson, 2017), não é impossível pensar que uma diversidade desconhecida de espécies possa ter existido no Pleistoceno.

O registro de Sylvilagus observado na Gruta Cuvieri aponta para sua existência no final do Pleistoceno, com desaparecimento no Holoceno inicial e reaparecimento em torno de 6 mil anos, no Holoceno médio.

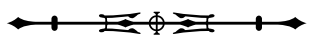


Apesar dessa ausência no registro da jazida, os Sylvilagus existiram em outras localidades ao Sul do Brasil até o limite do Holoceno inicial e médio e também persistiram do final do Pleistoceno até 8.500 AP no norte de Minas Gerais (Tabela 3). A variação da frequência no registro pode ser sugestiva de mudanças ambientais diferenciadas geograficamente durante o Holoceno. A presença de um período seco proposto por Araujo et al. (2005) na região de Lagoa Santa pode ter sido um limitador ambiental para o registro deste gênero nesta região entre 12.000 e 6.000 AP.

\section{AGRADECIMENTOS}

Agradecemos aos pesquisadores que coletaram o material da Gruta Cuvieri e que forneceram informações importantes para a realização deste trabalho. Ao revisor, Dr. Leonardo Kerber, pelas válidas contribuições. Agradecemos ao apoio financeiro dado aos autores: MO recebeu bolsa de produtividade do Conselho Nacional de Desenvolvimento Científico e Tecnológico (CNPq) (302163/2017-4) e auxílio à pesquisa Jovem Pesquisador da Fundação de Amparo à Pesquisa do Estado de São Paulo (JPFAPESP) (2018/23282-5), GSM, bolsa de doutorado pleno da Coordenação de Aperfeiçoamento de Pessoal de Nível Superior (CAPES) (88882.425678/2019-01).

\section{REFERÊNCIAS}

ALVES, M. A., A. GELLIS FILHO \& L. PELLARIN, 2005. Sítio de Água Limpa, Monte Alto, São Paulo: estruturas funerárias e avaliação radiológica de ossos humanos. Revista Canindé Xingó (5): 207-215.

AMEGHINO, F., 1907. Notas sobre una pequenia coleccion de huesos de mamíferos procedentes de las grutas calcareas de Iporanga en el Estado de São Paulo - Brazil. Revista do Museu Paulista 7: 59-124.

AraujO, A. G. M., W. A. NEVES, L. PILO \& J. P. ATUI, 2005. Holocene dryness and human occupation in Brazil during the "Archaic Gap". Quaternary Research 64(3): 298-307. DOI: https://doi. org/10.1016/j.yqres.2005.08.002

ARAUJO, A. G. M., L. B. PILÓ \& W. A. NEVES, 2010. Estratigrafia e processos de formação do sítio. In: A. G. M. ARAUJO \& W. NEVES (Org.): Lapa das Boleiras: um sítio paleoíndio do Carste de Lagoa Santa: 35-74. Ed. Annablume, São Paulo.
BADGLEY, C., 1986. Counting individuals in mammalian fossil assemblages from fluvial environments. Palaios 1(3): 338. DOI: https://doi.org/10.2307/3514695

BEHRENSMEYER, A. K., 1991. Terrestrial vertebrate accumulations. Taphonomy: In: P. A. ALLISON \& D. E. G. BRIGGS (Ed.): Taphonomy: releasing the data locked in the fossil record: 291-335. Plenum Press, New York.

BONVICINO, C. R., A. N. MENEZES, A. LAZAR, V. PENNAFIRME, C. BUENO, M. C. VIANA, P. S. D'ANDREA \& A. LANGGUTH, 2015. Chromosomes and phylogeography of Sylvilagus (Mammalia: Leporidae) from eastern Brazil. Oecologia Australis 19(1): 158-172. DOI: https://doi.org/10.4257/ oeco.2015.1901.10

BORGES, C., 2009. Analyse Archéozoologique d'un Amascoquillier Fluviatile: le Site Laranjal Vallée du Ribeira de Iguape - Etat de São Paulo - Brésil. Dissertação de Mestrado - Muséum National d'Histoire Naturelle, Paris.

CHAHUD, A., 2020a. Dasyproctidae e Cuniculidae (Cavioidea, Rodentia) do Pleistoceno da Gruta Cuvieri, estado de Minas Gerais, Brasil. Revista de Biologia e Ciências da Terra 20(1): 29-37.

CHAHUD, A., 2020b. Occurrence of the sabretooth cat Smilodon (Felidae, Machairodontinae) in the Cuvieri cave, eastern Brazil. Palaeontologia Electronica 23(2): a24. DOI: https://doi. org/10.26879/1056

CHAHUD, A., 2020c. Um exemplar muito jovem de Mazama sp. encontrado na Gruta Cuvieri, Região de Lagoa Santa, Estado de Minas Gerais, Brasil. Revista Brasileira de Zoociências 21(1): 1-10.

CHAHUD, A. \& M. OKUMURA, 2020a. The presence of Panthera onca Linnaeus 1758 (Felidae) in the Pleistocene of the region of Lagoa Santa, State of Minas Gerais, Brazil. Historical Biology. DOI: https://doi.org/10.1080/08912963.2020.1808975

CHAHUD, A. \& M. OKUMURA, 2020b. The youngest Tapir of a Quaternary deposit of the Americas. Historical Biology. DOI: https://doi.org/10.1080/08912963.2020.1798420

CHIM, E., 2018. Zooarqueologia da Lapa Grande de Taquaraçu. Dissertação de Mestrado - Museu de Arqueologia e Etnologia da Universidade de São Paulo, São Paulo.

DIERSING, V. E \& D. E. WILSON, 2017. Systematic status of the rabbits Sylvilagus brasiliensis and $S$. sanctaemartae from northwestern South America with comparisons to Central American populations. Journal of Mammalogy 98(6): 11641-1656. DOI: https://doi.org/10.1093/jmammal/gyx133

EMRY, R. J. \& C. E. GAWNE, 1986. A primitive, early Oligocene species of Palaeolagus (Mammalia, Lagomorpha) from the Flagstaff Rim area of Wyoming. Journal of Vertebrate Paleontology 6(3): 271-280. DOI: https://doi.org/10.1080/02 724634.1986 .10011622

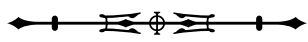


FICCARELLI, G., A. AZZAROLI, V. BORSELLI, M. COLTORTI, F. DRAMIS, O. FEJFAR, A. HIRTZ \& D. TORRE, 1992. Stratigraphical and paleontology of the Upper Pleistocene deposits in the Interandean Depression, Northern Ecuador. Journal of South America Earth Science 6(3): 145-150. DOI: https://doi. org/10.1016/0895-9811(92)90004-I

HADDAD-MARTIM, P. M., A. HUBBE, P. C. F. GIANNINI, A. S. AULER, L. B. PILÓ, M. HUBBE, E. MAYER, X. WANG, H. CHENG, R. L. EDWARDS \& W. A. NEVES, 2017. Quaternary depositional facies in cave entrances and their relation to landscape evolution: the example of Cuvieri Cave, eastern Brazil. Catena 157: 372-387. DOI: https://doi.org/10.1016/j.catena.2017.05.029

HERMENEGILDO, T., 2009. Reconstituição da dieta e dos padrões de subsistência das populações pré-históricas de caçadores coletores do Brasil central através da ecologia isotópica. Dissertação de Mestrado - Escola Superior de Agricultura Luiz de Queiroz, Universidade de São Paulo, Piracicaba.

HOCKETT, B. \& D. L. JENKINS, 2013. Identifying stone tool cut marks and the Pre-Clovis occupation of the Paisley Caves. American Antiquity 78(4): 762-778.

HOFFMAN, R. S. \& A. T. SMITH, 2005. Order Lagomorpha. In: D. E. WILSON \& D. M. REEDER (Ed.): Mammal species of the world: a taxonomic and geographic reference: 3. ed.: 194-211. Johns Hopkins University Press, Baltimore.

HOLZ, M. \& M. G. SIMÕES, 2002. Elementos fundamentais de Tafonomia: 1-231. Editora Universidade/UFRGS, Porto Alegre.

HUBBE, A., 2008. Contextualização taxonômica, tafonômica e morfométrica dos remanescentes ósseos da megamastofauna da gruta Cuvieri (MG), um sítio paleontológico do pleistoceno tardio. Dissertação de Mestrado - Universidade de São Paulo, São Paulo.

HUBBE, A., P. M. HADDAD-MARTIM, M. HUBBE, E. L. MAYER, A. STRAUSS, A. S. AULER, L. B. PILÓ \& W. A. NEVES, 2011. Identification and importance of critical depositional gaps in pitfall cave environments: the fossiliferous deposit of Cuvieri Cave, eastern Brazil. Palaeogeography, Palaeoclimatology, Palaeoecology 312(1-2): 66-78. DOI: https://doi.org/10.1016/j. palaeo.2011.09.010

IMAZIO DA SILVEIRA, M., 2001. "Você é o que você come": aspectos da subsistência no Sambaqui do Moa - Saquarema/RJ. Tese de Doutorado - Universidade de São Paulo, São Paulo.

JACOBUS, A. \& A. ROSA, 2013. Antigos habitantes do quadrante patrulhense e os animais. Pesquisas Antropologia (70): 241-254.

KIPNIS, R., 2002. Foraging societies of Eastern Central Brazil: an evolutionary ecology study of subsistence strategies during the terminal Pleistocene and Early/Middle Holocene. PhD Doctoral Dissertation - University of Michigan, Ann Arbor.
KIPNIS, R., M. BISSARO JR. \& H. M. PRADO, 2010. Os restos faunísticos. In: A. G. M. ARAUJO \& W. NEVES (Org.): Lapa das Boleiras: um sítio paleoíndio do Carste de Lagoa Santa: 121-147. Ed. Annablume, São Paulo.

LYMAN, R. L., 1994. Vertebrate Taphonomy: 1-552. Cambridge University Press (Cambridge Manuals in Archaeology), Cambridge. DOI: https://doi.org/10.1017/CBO9781139878302

MAYER, E. L., 2011. Processos de formação de um depósito fossilífero, um abismo na Gruta Cuvieri (MG): taxonomia, tafonomia e distribuição espacial - Dissertação de Mestrado, Universidade de São Paulo, São Paulo.

MINGATOS, G. S., 2017. Caça ou deixa passar? A dieta dos grupos humanos do sítio Lapa do Santo, Lagoa Santa, Minas Gerais. Dissertação de Mestrado - Universidade Federal do Rio de Janeiro, Rio de Janeiro.

MINGATOS, G. S. \& M. OKUMURA, 2016. Modelo de Amplitude de Dieta aplicada a restos faunísticos do sítio Lapa do Santo (MG) e suas implicações para o entendimento da dieta em grupos paleoíndios do Brasil central. Palaeoindian Archaeology 1(1): 15-31.

PAULA COUTO, C., 1979. Tratado de Paleomastozoologia: 1-590. Academia Brasileira de Ciências, Rio de Janeiro.

QUEIROZ, A. N., 2001. Contribution á l'étude archéozoologique des vertebres de cinq sites préhistoriques de trois régions du Brésil. Tese de Doutorado - Universite de Geneve, Suíça.

ROSA, A. O., 2009. Análise zooarqueológica do Sítio Garivaldino (RS-TA- 58), Município de Montenegro, RS. Pesquisas. Antropologia (67): 133-172.

ROSE, K. D., V. B. DELEON, P. MISSIAEN, R. S. RANA, A. SAHNI, L. SINGH \& T. SMITH, 2008. Early Eocene lagomorph (Mammalia) from western India and the early diversification of Lagomorpha. Proceedings Royal Society of London B 275(1639): 1203-1208. DOI: https://doi. org/10.1098/rspb.2007.1661

RUEDAS, L. A., 2017. A new species of cottontail rabbit (Lagomorpha: Leporidae: Sylvilagus) from Suriname, with comments on the taxonomy of allied taxa from northern South America. Journal of Mammalogy 98(4): 1042-1059. DOI: https://doi.org/10.1093/jmammal/gyx048

RUEDAS, L. A., S. M. SILVA, J. H. FRENCH, R. N. PLATT II, J. SALAZAR-BRAVO, J. M. MORA \& C. W. THOMPSON, 2017. A prolegomenon to the systematics of South American cottontail rabbits (Mammalia, Lagomorpha, Leporidae: Sylvilagus): designation of a neotype for S. brasiliensis (Linnaeus, 1758), and restoration of S. andinus (Thomas, 1897) and S. tapetillus Thomas, 1913": 1-67. University of Michigan, Museum of Zoology (Publications of the Museum of Zoology, 205), Ann Arbor.

VOORHIES, M., 1969. Taphonomy and population dynamics of an early Pliocene vertebrate fauna, Knox County, Nebraska. University of Wyoming (Contributions to Geology, 1), Laramie. DOI: https://doi.org/10.2113/gsrocky.8.special_paper_1.1

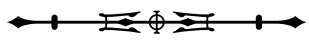


WAGUESPACK, N. M. \& T. A. SUROVELL, 2003. Clovis hunting strategies, or how to make out on plentiful resources. American Antiquity 68(2): 333-352. DOI: https://doi.org/10.2307/3557083

WINGE, H., 1887. Jordgundne og nulevende Gnawers (rodentia) fra Lagoa Santa, Minas Gerais, Brasilien. E Museo Lundii 3(1): 1-200.
WOODBURNE, M. O., 2010. The great American biotic interchange: dispersals, tectonics, climate, sea level and holding pens. Journal of Mammalian Evolution 17(4): 245-264. DOI: https://doi. org/10.1007/s10914-010-9144-8 